\title{
Induction of Antioxidant Metabolites in Moringa oleifera Callus by
}

\section{Abiotic Stress}

Letizia Zanella, Gabriele Di Marco, Angelo Gismondi, Roberto Braglia, Francesco Scuderi, Enrico L. Redi, Lorena Canuti, Andrea Galgani and Antonella Canini*

Department of Biology, University of Rome "Tor Vergata", via della Ricerca Scientifica 1, Rome - 00133, Italy.

\section{Corresponding Author}

*Tel (A. Canini): +39 067259 4333. Fax: +39 062025 300. E-mail: canini@ uniroma2.it 


\section{Table of content}

SI - Table S1. Concentrations of secondary metabolite in moringa callus exposed to different temperature stress intensity and duration................................................

SI - Table S2. Concentrations of secondary metabolite in moringa young leaves obtained by

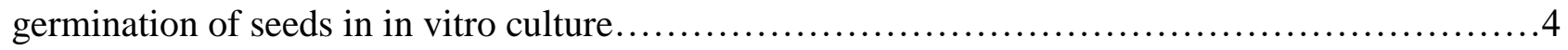

SI - Figure S1. Total phenol analysis performed on moringa callus exposed to different temperature stress intensity and duration (Cold stress, CS; hot stress, HS)....................................

SI - Figure S2. Antiradical activity of the extracts obtained from moringa callus exposed to different temperature stress intensity and duration. Results of FRAP (A) and ABTS (B) antioxidant assay were shown. Data were reported as $\mu \mathrm{g}$ AAE/g FW... 
SI - Table S1. Concentrations of secondary metabolite in moringa callus exposed to different temperature stress intensity and duration.

\begin{tabular}{|c|c|c|c|c|c|c|c|c|c|c|c|c|}
\hline & \multicolumn{2}{|c|}{ CS $6 \mathrm{~h}$} & \multicolumn{2}{|c|}{ CS $12 \mathrm{~h}$} & \multicolumn{2}{|c|}{ CS $24 \mathrm{~h}$} & \multicolumn{2}{|c|}{ CS 48 h } & \multicolumn{2}{|c|}{ CS 7 D } & \multicolumn{2}{|c|}{ HS } \\
\hline & control & treated & treated & control & control & treated & control & treated & control & treated & control & treated \\
\hline \multicolumn{13}{|l|}{ Phenolic acids } \\
\hline Gallic acid & $2.037 \pm 0.04$ & $0.71 \pm 0.32$ & $1.01 \pm 0.07$ & $2.52 \pm 0.03$ & $0.55 \pm 0.27$ & $1.62 \pm 0.21$ & $0.57 \pm 0.0005$ & $0.73 \pm 0.18$ & $1.13 \pm 0.29$ & $1.01 \pm 0.28$ & $0.72 \pm 0.72$ & $0.77 \pm 0.23$ \\
\hline Chlorogenic acid & $0.84 \pm 0.04$ & $1.5 \pm 0.27$ & $0.71 \pm 0.08$ & $0.63 \pm 0.06$ & $0.96 \pm 0.12$ & $0.76 \pm 0.04$ & $0.69 \pm 0.007$ & $1.26 \pm 0.35$ & $0.68 \pm 0.1$ & $0.56 \pm 0.1$ & $0.92 \pm 0.13$ & $2.46 \pm 0.73$ \\
\hline Caffeic acid & $0.79 \pm 0.22$ & $1.92 \pm 0.4$ & $1.39 \pm 0.13$ & $0.84 \pm 0.25$ & $1.13 \pm 0.19$ & $0.77 \pm 0.04$ & $0.72 \pm 0.005$ & $0.91 \pm 0.32$ & $0.78 \pm 0.11$ & $0.59 \pm 0.06$ & $1.16 \pm 0.23$ & $3.47 \pm 0.91$ \\
\hline$p$-Coumaric acid & $0.63 \pm 0.04$ & $0.67 \pm 0.1$ & $0.47 \pm 0.03$ & $0.6 \pm 0.06$ & $0.51 \pm 0.06$ & $0.55 \pm 0.03$ & $0.26 \pm 0.006$ & $0.32 \pm 0.04$ & $0.57 \pm 0.22$ & $0.44 \pm 0.02$ & $0.41 \pm 0.06$ & $1.59 \pm 1.21$ \\
\hline Phenyl caffeate & $2.78 \pm 0.14$ & $3.81 \pm 0.55$ & $2.87 \pm 0.26$ & $3.12 \pm 0.24$ & $2.83 \pm 0.35$ & $4.46 \pm 0.54$ & $2.07 \pm 0.02$ & $2.21 \pm 0.24$ & $2.78 \pm 0.36$ & $2.21 \pm 0.23$ & $2.69 \pm 0.41$ & $1.79 \pm 0.16$ \\
\hline \multicolumn{13}{|l|}{ Flavonoids } \\
\hline Rutin & $1.71 \pm 0.06$ & $1.89 \pm 0.1$ & $1.79 \pm 0.07$ & $1.66 \pm 0.08$ & $0.66 \pm 0.66$ & $1.57 \pm 0.24$ & $1.25 \pm 0.01$ & $1.41 \pm 0.13$ & $1.46 \pm 0.17$ & $0.44 \pm 0.3$ & $1.83 \pm 0.23$ & $0.93 \pm 0.25$ \\
\hline $\begin{array}{l}\text { 3-O- } \alpha \text {-L-arabinopyranosyl } \\
\text { quercetin }\end{array}$ & n.d. & $0.09 \pm 0.09$ & n.d. & n.d. & n.d. & n.d. & n.d. & n.d. & n.d. & n.d. & n.d. & n.d. \\
\hline Myricetin & $0.10 \pm 0.01$ & $0.10 \pm 0.03$ & $0.02 \pm 0.01$ & $0.11 \pm 0.01$ & $0.10 \pm 0.01$ & $0.04 \pm 0.02$ & $0.04 \pm 0.008$ & $0.06 \pm 0.01$ & $0.05 \pm 0.03$ & $0.02 \pm 0.007$ & $0.01 \pm 0.006$ & $0.04 \pm 0.03$ \\
\hline $\begin{array}{l}\text { 3-O- } \beta \text {-D-glucopyranosyl } \\
\text { kaempferol }\end{array}$ & $0.59 \pm 0.56$ & $0.77 \pm 0.37$ & n.d. & $0.56 \pm 0.52$ & $0.27 \pm 0.12$ & $0.06 \pm 0.03$ & n.d. & $0.52 \pm 0.03$ & $0.63 \pm 0.58$ & $0.16 \pm 0.06$ & n.d. & n.d. \\
\hline Genistein & n.d. & $0.02 \pm 0.02$ & $0.004 \pm 0.004$ & n.d. & $0.01 \pm 0.005$ & $0.01 \pm 0.005$ & n.d. & n.d. & $0.01 \pm 0.01$ & $0.07 \pm 0.02$ & n.d. & $0.0008 \pm 0.0006$ \\
\hline Kaempferol & $0.02 \pm 0.02$ & $0.01 \pm 0.01$ & n.d. & $0.02 \pm 0.03$ & $0.003 \pm 0.03$ & $0.001 \pm 0.001$ & n.d. & $0.04 \pm 0.02$ & n.d. & n.d. & n.d. & n.d. \\
\hline Apigenin & $0.03 \pm 0.03$ & $0.06 \pm 0.02$ & $0.02 \pm 0.01$ & $0.04 \pm 0.06$ & $0.07 \pm 0.07$ & $0.01 \pm 0.004$ & n.d. & $0.03 \pm 0.02$ & $0.001 \pm 0.0008$ & $0.01 \pm 0.008$ & n.d. & $0.0005 \pm 0.0005$ \\
\hline Chrysin & $0.04 \pm 0.04$ & $0.2 \pm 0.2$ & n.d. & $0.03 \pm 0.06$ & n.d. & $0.01 \pm 0.01$ & n.d. & n.d. & n.d. & $0.009 \pm 0.009$ & n.d. & $0.02 \pm 0.01$ \\
\hline Galangin & $0.03 \pm 0.02$ & $0.2 \pm 0.08$ & $0.003 \pm 0.0003$ & $0.03 \pm 0.02$ & $0.22 \pm 0.1$ & $0.07 \pm 0.02$ & $0.06 \pm 0.01$ & $0.13 \pm 0.04$ & $0.004 \pm 0.001$ & $0.12 \pm 0.05$ & $0.05 \pm 0.03$ & $0.01 \pm 0.006$ \\
\hline
\end{tabular}

Data were expressed as mean of three independent measurements \pm standard error. 
SI - Table S2. Concentrations of secondary metabolite in moringa callus young leaves obtained by germination of seeds in in vitro culture.

\begin{tabular}{|c|c|}
\hline MOLECULES & LEAF \\
\hline \multicolumn{2}{|l|}{ Phenolic acids } \\
\hline Gallic acid & n.d. \\
\hline Chlorogenic acid & $31.56 \pm 0.7$ \\
\hline Caffeic acid & $2.47 \pm 0.06$ \\
\hline$p$-Coumaric acid & $0.88 \pm 0.02$ \\
\hline 3,3-Dimethylallyl caffeate & n.d. \\
\hline Phenyl caffeate & $1.23 \pm 0.03$ \\
\hline \multicolumn{2}{|l|}{ Flavonoids } \\
\hline Rutin & $75.52 \pm 1.68$ \\
\hline $\begin{array}{l}\text { 3-O- } \alpha \text {-L-arabinopyranosyl } \\
\text { quercetin }\end{array}$ & $20.88 \pm 0.46$ \\
\hline Myricetin & $4.08 \pm 0.09$ \\
\hline $\begin{array}{l}\text { 3-O- } \beta \text {-D-glucopyranosyl } \\
\text { kaempferol }\end{array}$ & $7.39 \pm 0.16$ \\
\hline Quercetin & $2.73 \pm 0.06$ \\
\hline Genistein & $1.09 \pm 0.02$ \\
\hline Kaempferol & $0.84 \pm 0.02$ \\
\hline Apigenin & $4.15 \pm 0.09$ \\
\hline Chrysin & $1.28 \pm 0.03$ \\
\hline Galangin & $5.95 \pm 0.13$ \\
\hline
\end{tabular}

Data were expressed as mean of three independent measurements \pm standard error. 
SI - Figure S1. Total phenol analysis performed on moringa callus exposed to different temperature stress intensity and duration (Cold stress, CS; hot stress, HS).

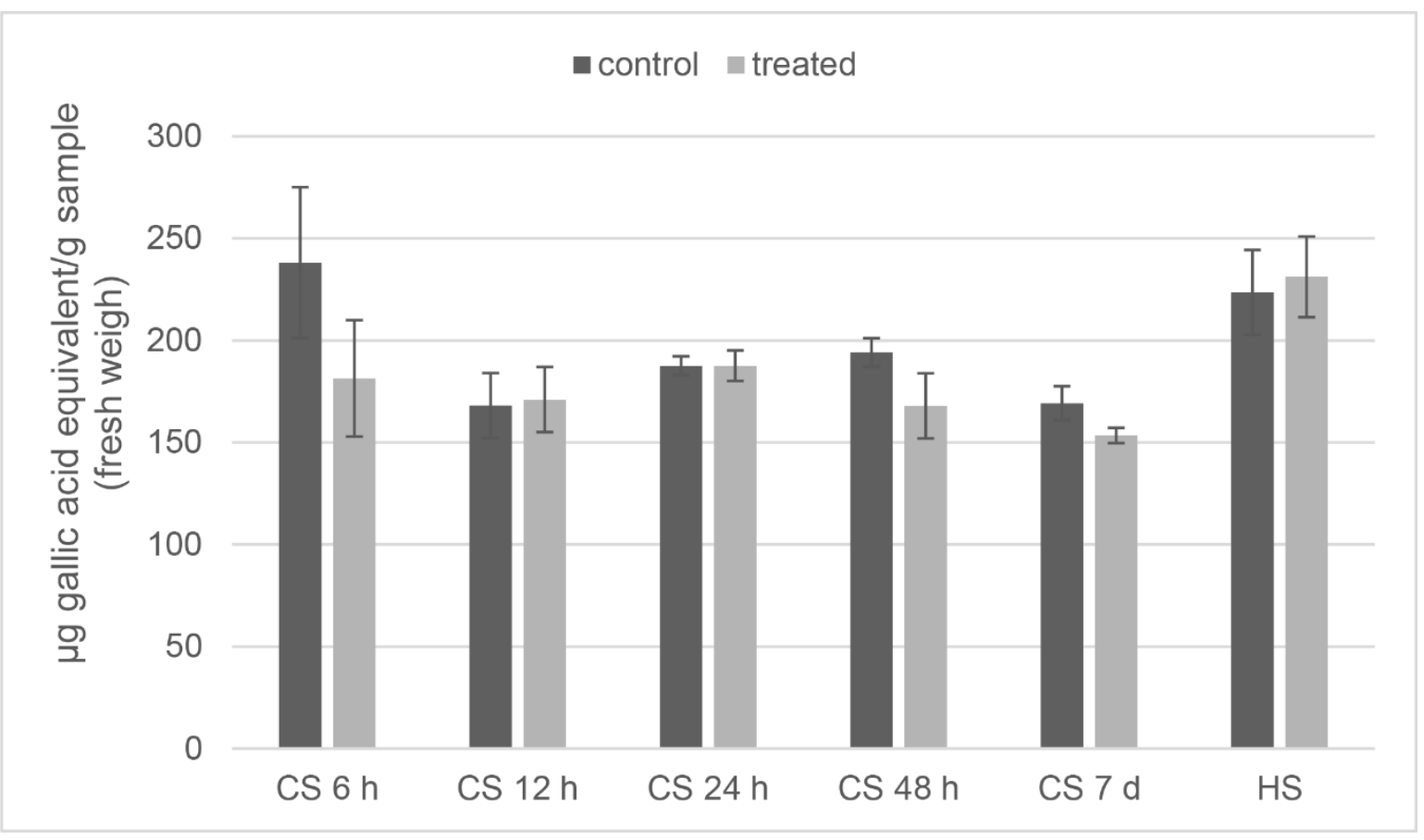


SI - Figure S2. Antiradical activity of the extracts obtained from moringa callus exposed to different temperature stress intensity and duration. Results of FRAP (A) and ABTS (B) antioxidant assay were shown. Data were reported as $\mu \mathrm{g}$ AAE/g FW.

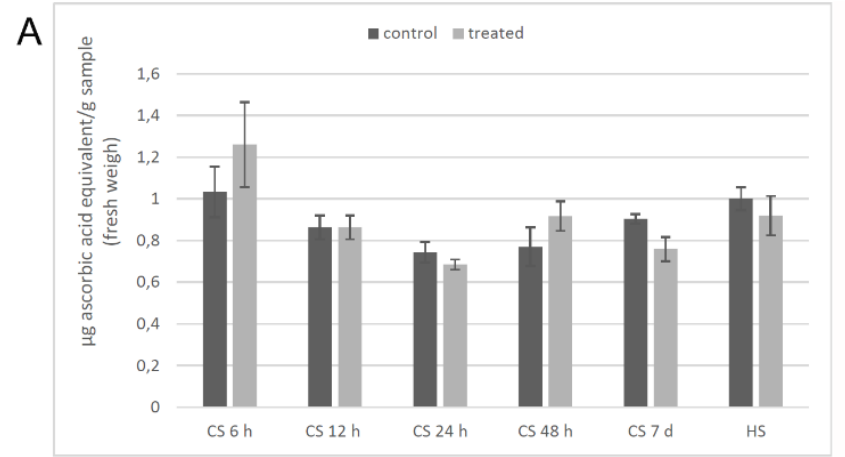

B

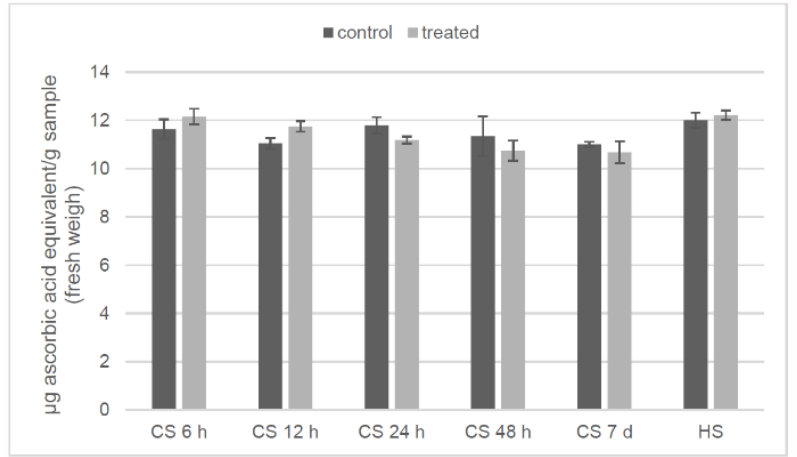

